\title{
Mathieu unit cell as a template for low emittance lattices
}

\author{
B. Riemann (1) \\ Paul Scherrer Institut, CH-5232 Villigen PSI, Switzerland
}

(Received 12 February 2020; accepted 25 February 2021; published 24 March 2021)

\begin{abstract}
The multibend achromat (MBA), which often serves as a building block for modern low-emittance storage rings, is composed of a repetition of unit cells with optimized optical functions for low emittance in the achromat center, as well as end cells for dispersion and optics matching to insertion devices. In this work, we describe the simplest stable class of unit cells that are based on a longitudinal Fourier expansion, transforming Hill equations to Mathieu equations. The resulting cell class exhibits continuously changing dipolar and quadrupolar moments along the beam path. Although this elementary model is defined by only three parameters, it captures a significant amount of notions that are applied in the design of MBAs. This is especially interesting as Mathieu cells can be viewed as an elementary extension of Christofilos' original model of alternating-gradient focusing, while their sinusoidal bending and focusing functions lend themselves to future applications in undulatorlike structures. Mathieu cells can be used to estimate the range of reasonable cell tunes and put an emphasis on the combination of longitudinal gradient bending and reverse bending, as well as on strong horizontal focusing to reach emittances lower than the classic theoretical minimum emittance cell. Furthermore, the lowest emittances in this model are accompanied by small absolute momentum compaction factors.
\end{abstract}

DOI: 10.1103/PhysRevAccelBeams.24.031601

\section{INTRODUCTION}

For practical reasons, the evolution of lattices for low-emittance synchrotron storage rings, including the double-bend [1], triple-bend [2], and quadruple-bend achromats [3], is mostly based on modeling them with discrete elements representing accelerator magnets. This also includes computations for the emittance minimum of a given periodic cell $[4,5]$.

Recent developments in the field of multibend achromats (MBAs) have shown that longitudinal gradients in magnet strength can significantly decrease emittance (e.g., [6-8]), and that reverse bends [9] (see also Veksler's suggestion in [10]) are necessary to fully exploit these longitudinal gradients $[11,12]$. It has also been known for a long time that combined-function magnets can help to decrease horizontal emittance by manipulating damping partitions (see e.g., [2,3]).

These facts can inspire to model the focusing and bending functions of the periodic lattice structure (i.e., the unit cell) directly, by a set of basis functions that are periodic in cell length, instead of using distinct elements to represent magnets. In principle, the type of basis function

Published by the American Physical Society under the terms of the Creative Commons Attribution 4.0 International license. Further distribution of this work must maintain attribution to the author(s) and the published article's title, journal citation, and DOI. can be selected in an arbitrary manner. E.g., in [13] steplike basis functions are used and truncated at a high order, and a particle-swarm based optimization is applied in the resulting high-dimensional parameter space.

The choice of sinusoidal basis functions is motivated in Sec. II-in essence, higher harmonics of the unit cell require stronger magnet pole-tip fields than lower harmonics, which is especially important for miniaturized magnet arrangements, where the lowest harmonics will dominate. This statement can be related to the common treatment of undulators, which usually starts with a description of the lowest harmonics (e.g., [14]).

The focusing functions for Mathieu cells, which we introduce in this work, contain the lowest possible order of such basis functions that yield stable solutions and are discussed in Sec. III. It is interesting to note that these sinusoidal focusing forces are also the starting point for Christofilos' description of alternating-gradient focusing [15]. However, his derivations focus on qualitative aspects of the motion, and not on solving the underlying differential equations - these are Mathieu equations.

Afterwards, bending functions are included in Sec. IV. The resulting parameter space is three-dimensional and can be explored without difficulty. For the resulting cells, synchrotron integrals, emittance and momentum compaction can be computed, and example solutions are studied.

The scaling laws for unit cells are investigated in Sec. V with an emphasis on the "chromaticity wall" and selecting the optimal cell length. A new objective function for the 
emittance of an arc with optimally scaled cell length is obtained, including constraints on applicable sextupole field strength. After further approximating the applicable pole-tip fields of magnets for a specific example tune, an example cell is constructed using parameters of the SLS 2.0 storage ring in Sec. VI.

\section{LONGITUDINAL HARMONICS}

Consider the magnetic field on a cylinder with radius $r$, and the beam path leading through its axis. For simplicity, we neglect the curvature of the path, although the argument naturally extends to that case. In a current-free region, a scalar potential defining the magnetic field $\vec{B}=-\nabla \Psi$ obeys the Laplace equation $\nabla^{2} \Psi=0$ [16]. In the aforementioned periodic cell, this potential can be expressed as linear combination of basis functions

$$
\tilde{\Psi}_{n, p}=D_{|n|}\left(\bar{k}_{p}, r\right) \mathrm{e}^{\mathrm{i} n \phi} \mathrm{e}^{\mathrm{i} \bar{k}_{p} z}
$$

for integers $p$ and $n$, and with the definition of

$$
D_{n}\left(\bar{k}_{p}, r\right)=2^{n} I_{n}\left(\bar{k}_{p} r\right) / \bar{k}_{p}^{n},
$$

where $I_{n}$ is the modified Bessel function of the first kind and order $n$ (see the Appendix A). Defining the period of the cell to be $L$, one obtains $\bar{k}_{p}=2 \pi p / L$.

When selecting a longitudinal harmonic with positive $n$, the radial field component at radius $r$ is given as (Appendix A)

$$
B_{r} \propto \frac{\mathrm{d} D_{n}}{\mathrm{~d} r} \propto r^{n-1}\left[1+\bar{k}_{p}^{2} \frac{n+2}{4 n(n+1)} r^{2}+\cdots\right] .
$$

For $p=0$ this reduces to the commonly known behavior $B_{r} \propto r^{n-1}$. The higher the longitudinal harmonic $|p|$ and thus the value of $\bar{k}_{p}$, the larger the term in square brackets, and the more difficult an application of the desired on-axis multipolar fields will become.

Therefore, lower longitudinal harmonics of multipolar fields are preferable to higher harmonics. Further assuming the unit cell to possess symmetry planes, we can select cosine functions $\cos \left(\bar{k}_{p} s\right)$ as basis functions with increasing positive order $p \leq P$.

\section{A. Biplanar stability}

The next task is finding the lowest maximum order $P$ for which stable particle motion could be achieved. The transverse linear motion of a charged particle with design energy in a decoupled accelerator lattice without bending magnets can be described using Hill differential equations $[17,18]$

$$
\begin{gathered}
\frac{\mathrm{d}^{2}}{\mathrm{~d} s^{2}} x(s)+\kappa(s) x(s)=0, \\
\frac{\mathrm{d}^{2}}{\mathrm{~d} s^{2}} y(s)-\kappa(s) y(s)=0 .
\end{gathered}
$$

Note that bending magnets (Sec. IV), chromatic effects (Sec. III A, Sec. V) and fringe effects (Sec. VC) are discussed in later sections.

Assuming $\kappa(s)$ to be constructed of basis functions $\cos \left(\bar{k}_{p} s\right)$, the most elementary case to consider is $P=0$ because then $\kappa=$ const. As the sign of $\kappa$ is different for the horizontal and vertical plane, bounded motion can only be achieved in one of them, and stable particle motion is impossible.

On the other hand, as we will see, the case $P=1$ already allows for stable motion. The resulting parameter space is low-dimensional, and thus lends itself to plain exploration. We first investigate such a model without bending and thus without dispersion. An additional parameter for bending is then included, and synchrotron radiation integrals (including damping partition, emittance, momentum compaction) are computable.

\section{MATHIEU EQUATIONS IN 2D}

To simplify the following calculations, we consider a normalized cell with the dimensionless length $\pi$. The normalized longitudinal cell coordinate $u$ is linked to the standard cell coordinate via $s=L u / \pi$ (see Appendix B).

Still considering the aforementioned focusing function for the case $P=1$, we obtain

$$
\begin{gathered}
\frac{\mathrm{d}^{2}}{\mathrm{~d} u^{2}} x(u)+k(u) x(u)=0, \\
\frac{\mathrm{d}^{2}}{\mathrm{~d} u^{2}} y(u)-k(u) y(u)=0
\end{gathered}
$$

with a cell-normalized focusing strength

$$
k(u)=k_{0}-2 k_{1} \cos (2 u)
$$

where the factor -2 was selected arbitrarily for alignment with standard notation. The equations of motion are now Mathieu equations, both depending on the same set of parameters $k_{0}, k_{1}$.

We analyze the horizontal motion based on Floquet solutions, mainly following the approach outlined in [19]. These can be written in the normal form [20]

$$
X(u)=\mathrm{e}^{\mathrm{i} 2 \nu_{\mathrm{x}} u} f(u),
$$

where $2 \nu_{\mathrm{x}}$ is the characteristic exponent and $\nu_{\mathrm{x}}$ is the horizontal cell tune, i.e., the betatron phase advance in a cell divided by $2 \pi$. We express the $\pi$-periodic function 


$$
f(u)=\sum_{q=-Q}^{Q} f_{q} \mathrm{e}^{\mathrm{i} 2 q u}
$$

as a truncated Fourier series with the highest harmonic being $Q$. For the following calculations, $Q=50$ is sufficient. Then using the $f_{q}$ as components of a vector $\vec{f}$, we can write Eq. (5) as

$$
0=\mathbf{M}\left(\nu_{\mathrm{x}}\right) \vec{f},
$$

where $\mathbf{M}\left(\nu_{\mathrm{x}}\right)$ is a (truncated) tridiagonal matrix of size $(2 Q+1) \times(2 Q+1)$ with entries

$$
M_{q, q}=1, \quad M_{q, q-1}=M_{q, q+1}=\frac{k_{1}}{4\left(q+\nu_{\mathrm{x}}\right)^{2}-k_{0}} .
$$

To solve this system, we require prior knowledge of $\nu_{\mathrm{x}}$. This can be achieved using the determinant of $\mathbf{M}\left(\nu_{\mathrm{x}}=0\right)$ and the Whittaker-Hill formula $[19,21]$

$$
\sin ^{2}\left(\pi \nu_{\mathrm{x}}\right)=C\left(k_{0}, k_{1}\right)
$$

with the definition

$$
C\left(k_{0}, k_{1}\right)=\operatorname{det} \mathbf{M}\left(\nu_{\mathrm{x}}=0\right) \cdot \sin ^{2}\left(\pi \sqrt{k_{0}} / 2\right) .
$$

It is apparent from Eq. (11) that periodic solutions only exist for

$$
0<C\left(k_{0}, k_{1}\right)<1,
$$

leading to limited regions in $\left(k_{0}, k_{1}\right)$ space where horizontally stable motion occurs. Furthermore, it follows from Eq. (5) that stability of vertical motion is equivalent to that of horizontal motion when mirroring the $\left(k_{0}, k_{1}\right)$ regions at the origin. The intersection of stability regions for both planes leads to islands of stability for transverse motion (see Fig. 1, cf. [[21] Fig. 5]).

The islands differ significantly in the maximal focusing strength that needs to be applied. The only stable solutions with reasonable $\max |k(u)| \leq 2$ all occur in a single stability island. This 'neck-tie' island, named here in analogy to the corresponding diagram for the FODO lattice [22] is shown in Fig. 2 in more detail. We conclude that reasonable cell designs require $\left(k_{0}, k_{1}\right)$ in this island, which has cell tunes $\nu_{\mathrm{x}}, \nu_{\mathrm{y}}<1 / 2$.

\section{A. Tune map for chromaticity}

As there exists a bijective mapping of stable-motion quadrupole configurations to tunes $\left(k_{0}, k_{1}\right) \leftrightarrow\left(\nu_{\mathrm{x}}, \nu_{\mathrm{y}}\right)$, we are able to study the properties of Mathieu unit cells directly in tune space.

Given $\nu_{\mathrm{x}}$, one may solve Eq. (9) for $f(u)$. Dimensionless optical functions in the unit cell of length $\pi$ are computed from $X(u)$ in Eq. (7) as (see, e.g., [23])

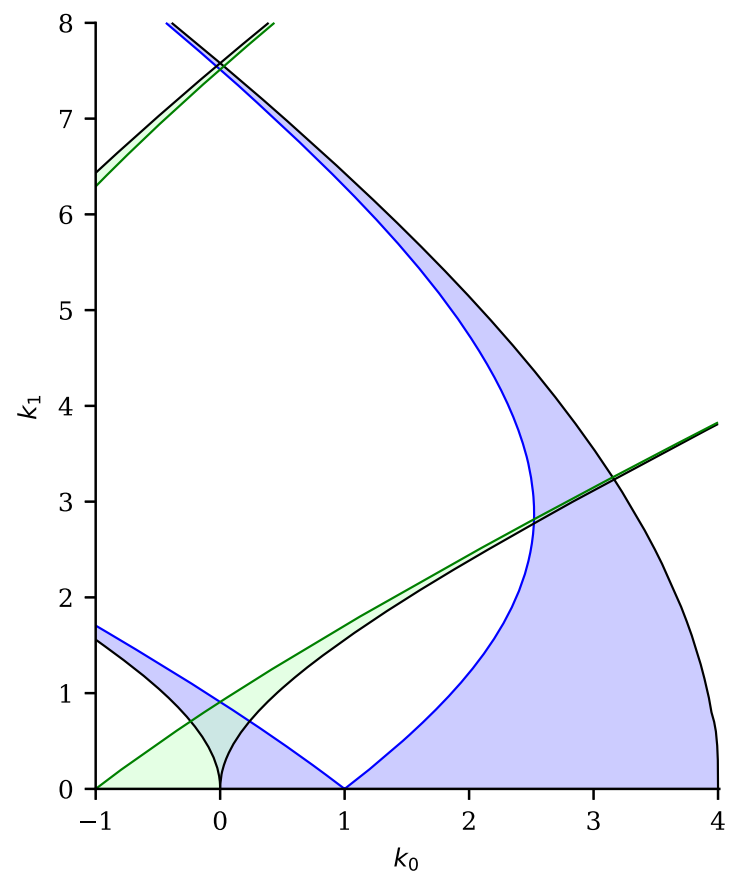

FIG. 1. Stability diagram in the $\left(k_{0}, k_{1}\right)$ plane. Blue-shaded regions are stable for horizontal motion, with the blue line indicating $\nu_{\mathrm{x}}=0.5$. Green-shaded regions are stable for vertical motion, with the green line indicating $\nu_{\mathrm{y}}=0.5$. The region overlaps are stability islands.

$$
\tilde{\beta}_{\mathrm{x}}(u)=X(u) X^{*}(u) / I_{\mathrm{x}}=f(u) f^{*}(u) / I_{\mathrm{x}}
$$

with

$$
I_{\mathrm{x}}=\Im\left\{X^{*}(u) X^{\prime}(u)\right\}=\Im\left\{f^{*}(u) f^{\prime}(u)\right\},
$$

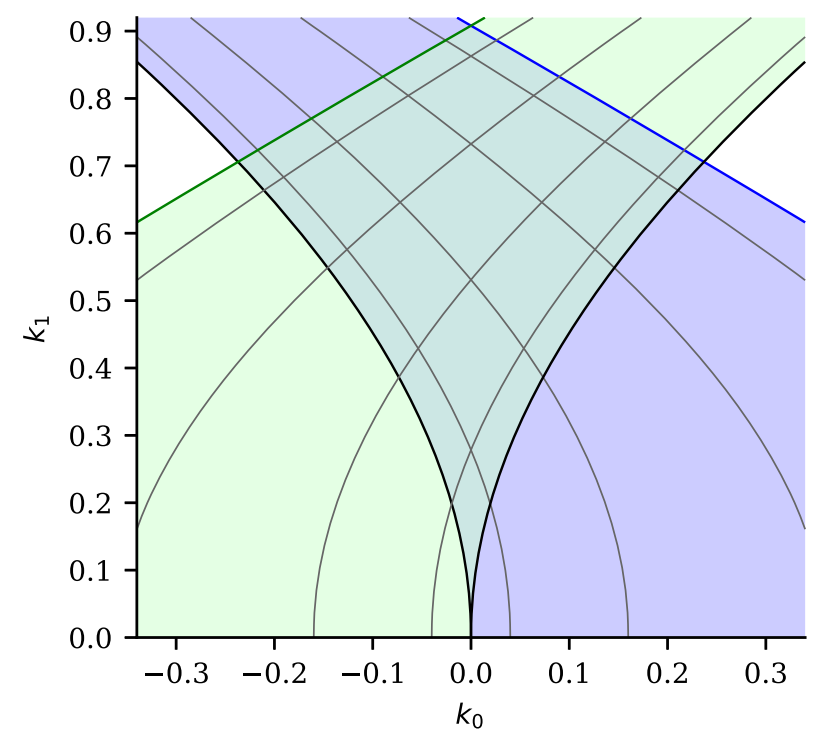

FIG. 2. Stability diagram for the neck-tie island. Shading and colored lines like in Fig. 1. Gray lines are isolines of $\nu_{\mathrm{x}}$ or $\nu_{\mathrm{y}}$ in steps of 0.1 . 
and they can be used to compute the linear chromaticity with the horizontal and vertical optical functions $\tilde{\beta}_{\mathrm{x}}, \tilde{\beta}_{\mathrm{y}}$ by (cf. [14])

$$
\begin{aligned}
& 4 \pi \xi_{\mathrm{x}}=-\int_{0}^{\pi} \tilde{\beta}_{\mathrm{x}}(u) k(u) \mathrm{d} u, \\
& 4 \pi \xi_{\mathrm{y}}=\int_{0}^{\pi} \tilde{\beta}_{\mathrm{y}}(u) k(u) \mathrm{d} u .
\end{aligned}
$$

Following from the aforementioned symmetry of vertical and horizontal motion in $\left(k_{0}, k_{1}\right)$ space, we obtain the vertical chromaticity for a given tune as

$$
\xi_{\mathrm{y}}\left(\nu_{\mathrm{x}}, \nu_{\mathrm{y}}\right)=\xi_{\mathrm{x}}\left(\nu_{\mathrm{y}}, \nu_{\mathrm{x}}\right) .
$$

The results of the linear chromaticity computation are shown in Fig. 3. In the usable regions of the tune map, i.e., considering stop-bands around the half-integer resonances, we obtain negative chromaticities $\xi_{\mathrm{x}, \mathrm{y}}>-2.5$.

Note that the general dependency of cell tune on particle energy - without effects by higher-order multipoles yet to be introduced - can be obtained by scaling the $\left(k_{0}, k_{1}\right)$ vector corresponding to a given tune in the neck-tie diagram in Fig. 2.

\section{BENDING AND EMITTANCE}

The next task is to include bending into the unit cell. We assume that the curvature is sufficiently small so that we can neglect the effect of weak focusing on $k(s)$ in Eq. (5). Following the same line of reasoning we also neglect edge focusing.

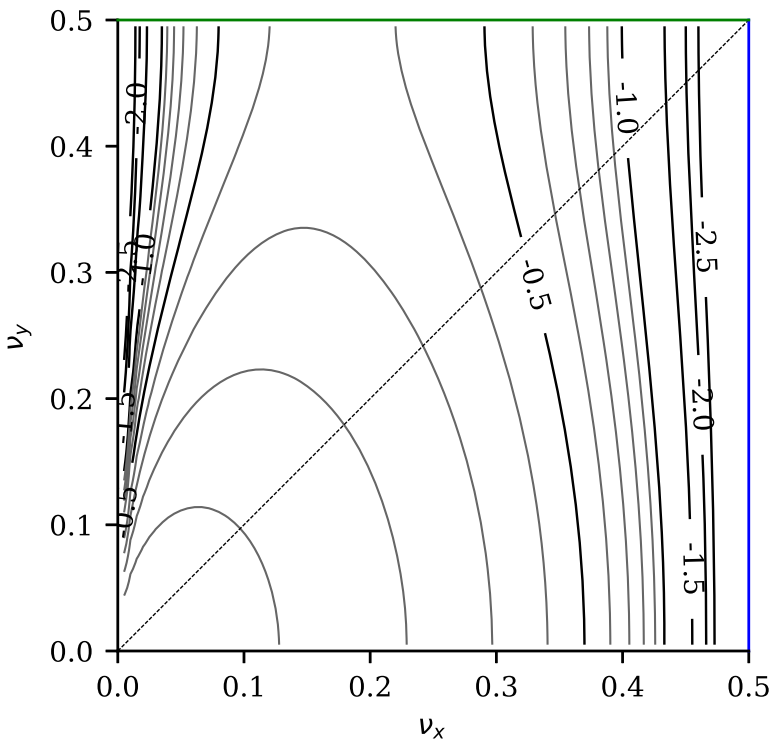

FIG. 3. Tune map for horizontal linear chromaticity. The borders of the neck-tie island are shown as colored edges of the plot, corresponding to the lines in Fig. 2.
The average curvature $\langle 1 / \rho\rangle$ in a cell is defined by the arc geometry of the storage ring and the bending of endcells. When assuming the curvature to contain low-order longitudinal harmonics in the same manner as the focusing strengths $(P=1)$, we can parametrize

$$
1 / \rho(u)=\langle 1 / \rho\rangle b(u)
$$

with a cell-normalized dipole strength

$$
b(u)=1-2 b_{1} \cos (2 u) .
$$

Note that $b(u)$ shares the same mirror symmetry around $u=0$ and $u=\pi / 2$ as $k(s)$, as this is the most elementary approach.

Also, an upper limit on $|b|$, and thus $\left|b_{1}\right|$, exists given by achievable dipole field strength independent of cell length, as

$$
\max |b|=\frac{\max |B|}{B_{\mathrm{c}}} .
$$

Here we introduced the characteristic magnetic field density

$$
B_{\mathrm{c}}=(B \rho)\langle 1 / \rho\rangle
$$

depending on the beam rigidity $(B \rho)$.

Normalizing with the average curvature, the inhomogeneous Hill equation for linear dispersion $\eta(s)[14,22]$ can be rewritten as (see Appendix B)

$$
\frac{\mathrm{d}^{2}}{\mathrm{~d} u^{2}} \tilde{\eta}(u)+k(u) \tilde{\eta}(u)=b(u)
$$

with

$$
\tilde{\eta}(u)=\left(\frac{\pi}{L}\right)^{2} \frac{\eta(u)}{\langle 1 / \rho\rangle} .
$$

We recognize that the solutions $\tilde{\eta}(u)$ of Eq. (22) are additive in $b(u)$. Let $\tilde{\eta}^{(0)}(u)$ be the solution for $b(u)=1$ and let $\tilde{\eta}^{(1)}(u)$ be the solution for $b(u)=-2 \cos (2 u)$. Then the general solution is linear in $b_{1}$, as

$$
\tilde{\eta}(u)=\tilde{\eta}^{(0)}(u)+b_{1} \tilde{\eta}^{(1)}(u) .
$$

The driving term $b(u)$ requires $\tilde{\eta}(u)$ to be periodic in $\pi$,

$$
\tilde{\eta}(u)=v_{0}+2 \sum_{q=1}^{Q} v_{q} \cos (2 q u)=\sum_{q=-Q}^{Q} v_{q} \mathrm{e}^{2 \mathrm{i} q u},
$$

so that Eq. (22) reduces to the solvable linear equation system 


$$
\mathbf{M}\left(\nu_{\mathrm{x}}=0\right) \vec{v}=\vec{c}
$$

with $c_{0}=1 / k_{0}, \quad c_{1}=c_{-1}=b_{1} /\left(4-k_{0}\right)$ and all other components of $\vec{c}$ being zero. The solution $\tilde{\eta}(u)$ can then be constructed using $\vec{v}$.

\section{A. Synchrotron integrals}

Having introduced bending and dispersion, knowledge of linear momentum compaction can be obtained, which is proportional to the synchrotron integral $[24,25]$ for the unit cell

$$
I_{1}=\int_{0}^{\pi} b(u) \tilde{\eta}(u) \mathrm{d} u
$$

To gain some insight into the behavior of $I_{1}$, we insert Eq. (24) and obtain

$$
\begin{aligned}
I_{1}\left(b_{1}\right)= & \int_{0}^{\pi} \tilde{\eta}^{(0)}(u) \mathrm{d} u \\
& +b_{1}\left[\int_{0}^{\pi} \tilde{\eta}^{(1)}(u) \mathrm{d} u-2 \int_{0}^{\pi} \tilde{\eta}^{(0)}(u) \cos (2 u) \mathrm{d} u\right] \\
& -b_{1}^{2} \int_{0}^{\pi} \tilde{\eta}^{(1)}(u)[1+\cos (4 u)] \mathrm{d} u .
\end{aligned}
$$

By its definition preceding Eq. (24) and due to symmetry conditions,

$$
\int_{0}^{\pi} \tilde{\eta}^{(1)} \mathrm{d} u=0
$$

Although $\tilde{\eta}(u)$ is the solution of a parametric oscillator, we may expect it to mainly oscillate at the driving frequency $\cos (2 u)$, making the last coefficient in Eq. (28) small.

We proceed by computing radiation properties for the normalized cell. The synchrotron integrals related to radiation loss and damping partitions are [24,25]

$$
I_{2}=\int_{0}^{\pi} b(u)^{2} \mathrm{~d} u=\pi\left(1+2 b_{1}^{2}\right)
$$

and

$$
I_{4} \approx 2 \int_{0}^{\pi} b(u) k(u) \tilde{\eta}(u) \mathrm{d} u .
$$

The expression used for $I_{4}$ is an approximation in which, in consistence with our assumption, the contribution of weak focusing has been omitted. In full analogy to $I_{1}$ and substituting $\tilde{\eta}^{(\cdot)} \rightarrow k \tilde{\eta}^{(\cdot)}$, we find that $I_{4}\left(b_{1}\right)$ is also a quadratic function of $b_{1}$.

In order for a flat lattice to allow damping in all dimensions, the horizontal damping partition

$$
J_{\mathrm{x}}=1-\frac{I_{4}}{I_{2}}
$$

must fulfill $0<J_{\mathrm{x}}<3[24,25]$.

In low-emittance rings, $J_{\mathrm{x}}>1$ is favored [26] as the effects of quantum excitation are then shifted from the transverse into the longitudinal plane.

The dispersion action $\mathcal{H}(s)$ occurring in the quantum excitation integral

$$
\mathcal{I}_{5}=\int_{0}^{\pi} \mathcal{H}(u)\left|b(u)^{3}\right| \mathrm{d} u
$$

can be computed using the Floquet solution as

$$
\begin{aligned}
\mathcal{H}(u) & =\tilde{\gamma} \tilde{\eta}^{2}+2 \tilde{\alpha} \tilde{\eta} \tilde{\eta}^{\prime}+\tilde{\beta} \tilde{\eta}^{\prime 2} \\
& =\left|\mathcal{X}^{\prime}(u) \tilde{\eta}(u)-X(u) \tilde{\eta}^{\prime}(u)\right|^{2} / I_{\mathrm{x}} .
\end{aligned}
$$

One can then obtain the emittance $\epsilon \propto I_{5} /\left(I_{2} J_{\mathrm{x}}\right)$. However, we are interested in the emittance relative to that of a normalized theoretical minimum emittance (TME) cell [5],

$$
F\left(\nu_{\mathrm{x}}, \nu_{\mathrm{y}}, b_{1}\right)=\frac{I_{5}}{I_{2} J_{\mathrm{x}}} /\left(\frac{I_{5}}{I_{2}}\right)_{\mathrm{TME}}=\frac{12 \sqrt{15}}{\pi^{3}} \frac{I_{5}}{I_{2} J_{\mathrm{x}}},
$$

as it is independent of cell length.

\section{B. Results}

We can now search for the optimal $b_{1}$ parameter to reach minimum emittance ratio $F$ for a given tune $\left(\nu_{\mathrm{x}}, \nu_{\mathrm{y}}\right)$; the results are shown in Fig. 4. Sub-TME emittances are reached for $0.4<\nu_{\mathrm{x}}<0.5$, with a minimal $F<0.7$. We see that, in this band, increasing $\nu_{\mathrm{y}}$ only has slight effectsincreasing $J_{\mathrm{x}}$ and decreasing $F$. Damping partitions for the sub-TME region are in a feasible interval $J_{\mathrm{x}} \in[1.5,2.5]$.

The region with small absolute momentum compaction in Fig. 4 has a similar location and shape as that of subTME emittance- - this is consistent with the general observation that low-emittance lattices require small absolute momentum compaction.

To further investigate the influence of the dipole coefficient $b_{1}$, which is not visible in the projections in Fig. 4, figures of merit for an example tune $\nu_{\mathrm{x}}=0.45, \nu_{\mathrm{y}}=0.35$ and a range of $b_{1}$ are shown in Fig. 5. According to Eq. (28) we expect $I_{1}$ to be quadratic in $b_{1}$, with the quadratic coefficient almost vanishing - we obtain a visibly linear dependency here. The location of $I_{1}=0$ and the location of the minimal $F$ again illustrate that low emittances and low momentum compaction are closely related.

As the damping partition is in a usable range, the minimum emittance solution for this tune is feasible. The example solution parameters, figures of merit, and optical functions are shown in Fig. 6 and Table I. It can be seen that (1) positive bending and defocusing quadrupole 


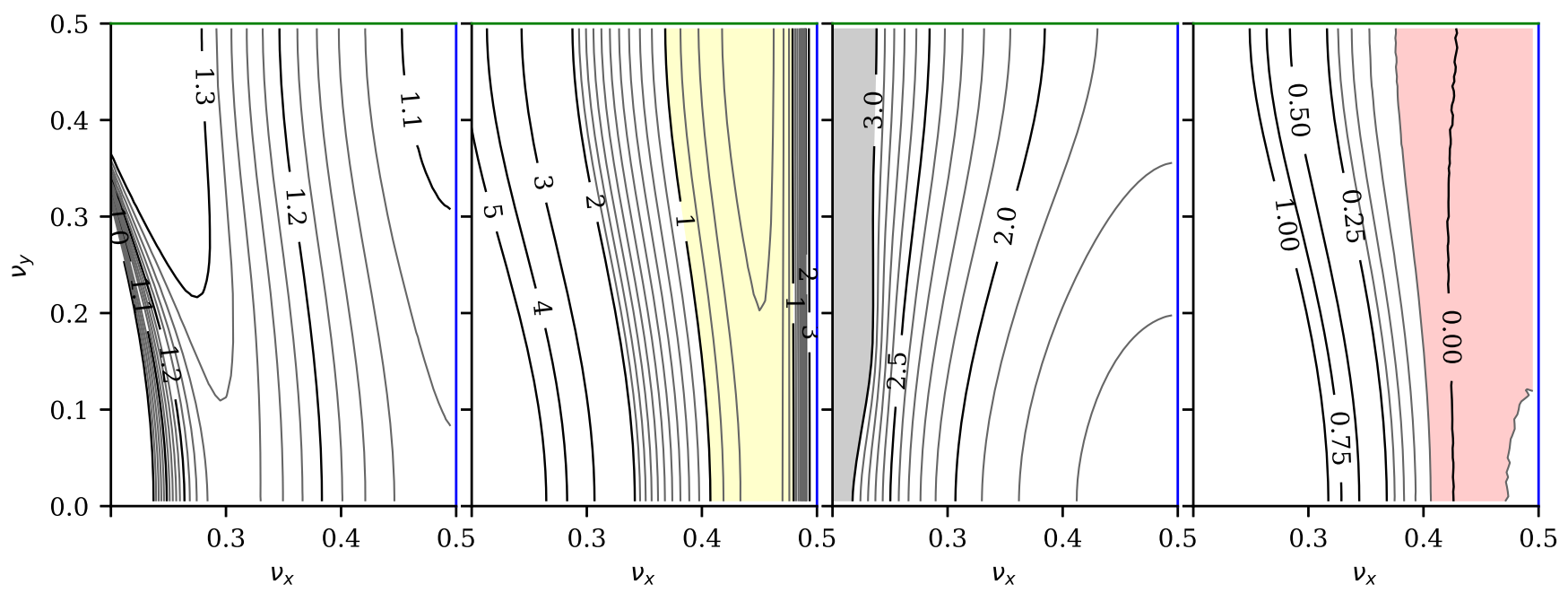

FIG. 4. From left to right: (1) optimized $b_{1}$ for minimal TME emittance ratio $F$ for $b_{1}<1.3$, (2) resulting $F$ up to 5, with sub-TME region in yellow, (3) horizontal damping partition $J_{\mathrm{x}}$, invalid region in gray, (4) momentum compaction integral $I_{1}$ up to 1 , with the red area indicating $\left|I_{1}\right|<0.05$.

fields overlap, increasing $J_{\mathrm{x}}$ [3], and that (2) reverse bending occurs at the position of maximum dispersion [11].

\section{SEXTUPOLES AND CHROMATICITY WALL}

To control linear chromaticity occurring according to Eq. (16), the introduction of sextupolar fields

$$
\mu(s)=\frac{1}{2(B \rho)} \frac{d^{2} B_{y}}{d x^{2}}(x=0, s),
$$

is required. Full compensation leads to the condition [22]

$$
\begin{aligned}
& 4 \pi \xi_{\mathrm{x}} \stackrel{!}{=}-\int \beta_{x}(s) \eta_{x}(s) \mu(s) \mathrm{d} s, \\
& 4 \pi \xi_{\mathrm{y}} \stackrel{!}{=} \int \beta_{y}(s) \eta_{x}(s) \mu(s) \mathrm{d} s .
\end{aligned}
$$

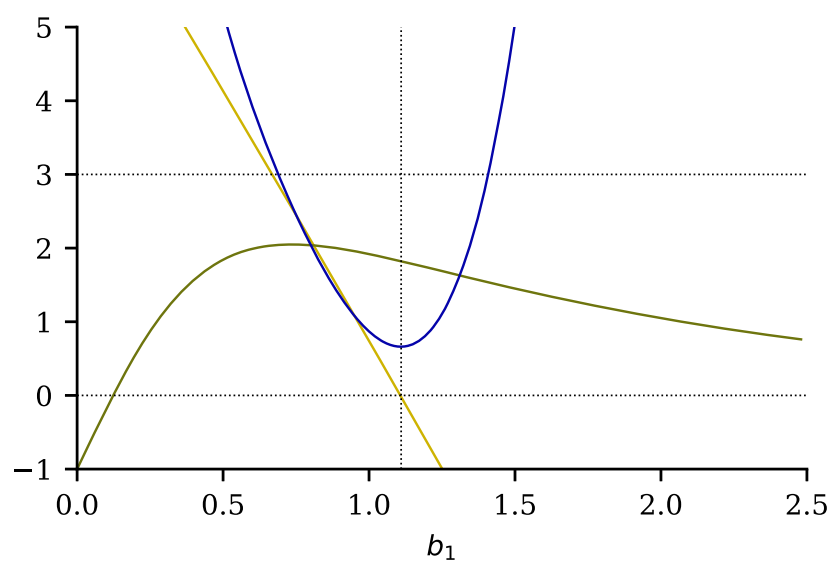

FIG. 5. TME emittance ratio $F$ (blue), horizontal damping partition $J_{\mathrm{x}}$ (green) and momentum compaction integral $I_{1}$ (yellow) in dependence of the dipole coefficient $b_{1}$. The limits of $J_{\mathrm{x}}$ for stable motion, as well as the $b_{1}$ value used in the example solution, are denoted with dashed lines.
We define a cell-normalized sextupolar field function

$$
m(u)=m_{0}+2 m_{1} \cos (2 u),
$$

which includes the fundamental harmonic and shares the same mirror symmetry as $b(u)$ and $k(u)$, yielding the most elementary mode.

For the unit cell with length $\pi$, this results (Appendix B) in an equation system

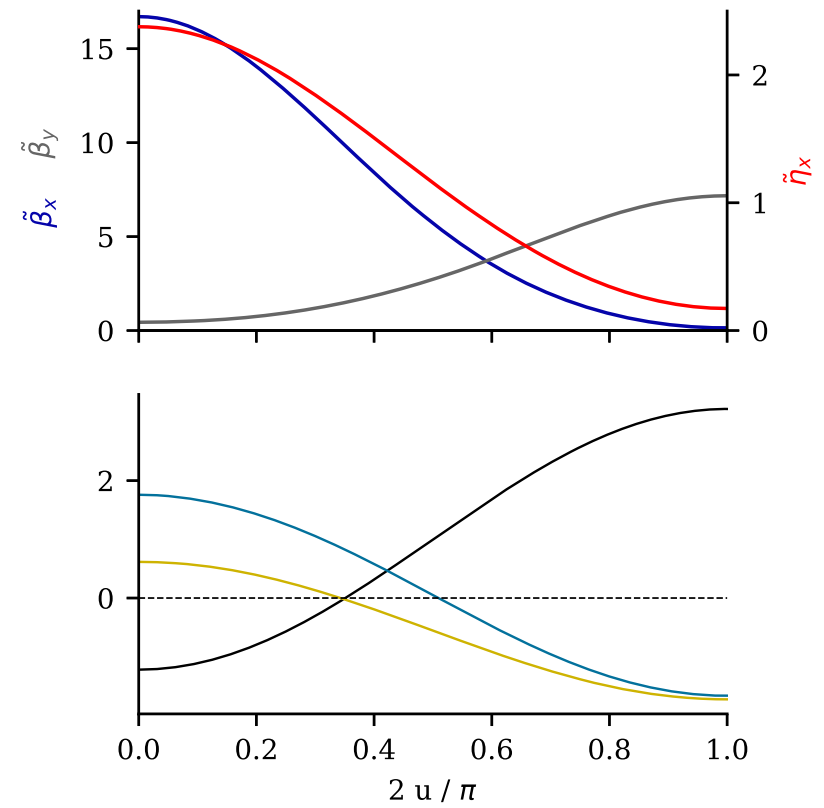

FIG. 6. Top: optical functions for the example solution characterized in Table I. Bottom: corresponding distribution of dipole (black), quadrupole (blue), and sextupole fields (yellow). 
$\xi_{\mathrm{x}}=-\frac{1}{2}\left\langle\tilde{\beta}_{x} \tilde{\eta} m\right\rangle=-\frac{\left\langle\tilde{\eta} \tilde{\beta}_{\mathrm{x}}\right\rangle}{2} m_{0}-\left\langle\tilde{\eta} \tilde{\beta}_{\mathrm{x}} \cos (2 u)\right\rangle m_{1}$,

$\xi_{\mathrm{y}}=\frac{1}{2}\left\langle\tilde{\beta}_{y} \tilde{\eta} m\right\rangle=\frac{\left\langle\tilde{\eta} \tilde{\beta}_{\mathrm{y}}\right\rangle}{2} m_{0}+\left\langle\tilde{\eta} \tilde{\beta}_{\mathrm{y}} \cos (2 u)\right\rangle m_{1}$,

which can be uniquely solved for $m_{0}, m_{1} ;\langle\cdot\rangle$ denotes the average of the respective quantity over the cell length. The sextupole coefficients for $F$-optimized cells with given cell tunes are shown in Fig. 7 and also included for the example in Table I.

\section{A. Sextupole-limited arc emittance}

We want to find the cell length yielding the optimal emittance for a given limited sextupole strength max $|m|$. In an arc of constant average curvature $\langle 1 / \rho\rangle$, the actual sextupole strength $\mu(u)$ scales relative to the sextupole strength of the normalized cell $m(u)$ as (see Appendix B)

$$
\mu(u)=\left(\frac{\pi}{L}\right)^{4} \frac{m(u)}{\langle 1 / \rho\rangle} .
$$

This disadvantageous dependency on cell length is sometimes referred to as "chromaticity wall" [27] and is a major limitation for shrinking unit cells.

The optimal cell length can be obtained from the above equation as

$$
L=\pi\left(\frac{\max |m|}{\max |\mu|\langle 1 / \rho\rangle}\right)^{1 / 4}
$$

It is well known (e.g., [28]) that the emittance scales with the cube of bending angle per cell, and thus in our case $\propto L^{3}$. Reusing the definition of TME-normalized emittance $F$ in Eq. (35), we find that the optimal emittance scales as

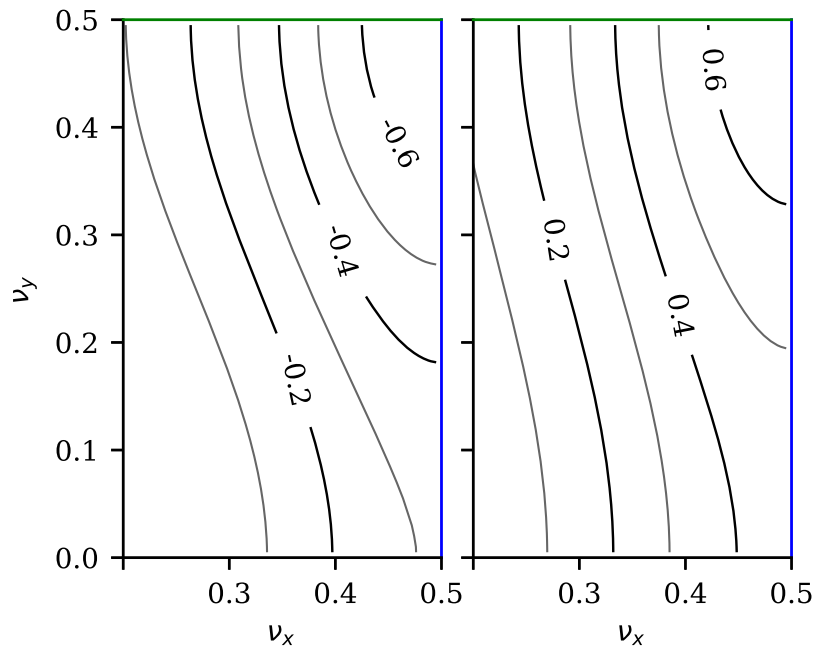

FIG. 7. Values of $m_{0}$ (left) and $m_{1}$ (right) for optimized emittance ratio $F$ at a given cell tune.
TABLE I. Example multipole parameters of a Mathieu cell and its figures of merit.

\begin{tabular}{lcc}
\hline \hline Parameter & & Value \\
\hline Dipole coefficient & $b_{1}$ & -1.1100 \\
Quadrupole coefficient & $k_{0}$ & 0.04801 \\
& $k_{1}$ & 0.8554 \\
Sextupole coefficient & $m_{0}$ & -0.5550 \\
& $m_{1}$ & 0.5855 \\
Horizontal cell tune & $\nu_{\mathrm{x}}$ & 0.4500 \\
Vertical cell tune & $\nu_{\mathrm{y}}$ & 0.3500 \\
Nat. horizontal cell chromaticity & $\xi_{\mathrm{x}}$ & -1.8323 \\
Nat. vertical cell chromaticity & $\xi_{\mathrm{y}}$ & -0.6716 \\
Horizontal damping partition & $J_{\mathrm{x}}$ & 1.8219 \\
Radiation integral & $I_{1}$ & -0.0191 \\
TME ratio & $F$ & 0.6617 \\
Arc emittance factor (sec. V) & $G$ & 0.9963 \\
\hline \hline
\end{tabular}

$$
\epsilon \propto F L^{3} \propto G, \quad \text { with } \quad G=F(\max |m|)^{3 / 4} .
$$

We can use $G$ as an objective function for optimization, thus including sextupolar fields in a straightforward manner, to find an optimal value for $b_{1}$.

Tune maps for figures of merit in which $b_{1}$ is selected to yield the optimal $G$ are shown in Fig. 8. We can observe that the characteristics for the emittance ratio $F$ and the damping partition $J_{\mathrm{x}}$ did not change significantly, although the tune-space region of low momentum compaction has reduced in size.

Furthermore, it is interesting that the two regions with $G \leq 1$ exist. One region has a significantly reduced horizontal focusing $\nu_{\mathrm{x}}<0.2$. Unfortunately, the low $G$ values in this region are mainly influenced by a large and infeasible damping partition $J_{\mathrm{x}}>3$ (see Fig. 9).

The other region overlaps with the low-emittance regime shown in Fig. 4, with the difference that there is now a slight preference for less vertical focusing. The additional parameters $G$ and $\max |m|$ for our example configuration, which is located in that region (see also Table I and Fig. 5), are shown in Fig. 10.

\section{B. Extensions to higher harmonics}

Having obtained an optimized solution for the Mathieu cell $(P=1)$, it is possible to iteratively increase $P$ and reoptimize the solution locally. However, the number of free parameters increases significantly. In the scope of this work, we increase to $P=2$ only for the sextupolar field component, so that

$$
m(u)=m_{0}+2 m_{1} \cos (2 u)+2 m_{2} \cos (4 u) .
$$

This has the advantage that the dimensions of the free parameter space $\left(\nu_{\mathrm{x}}, \nu_{\mathrm{y}}, b_{1}\right)$ do not increase- the additional 


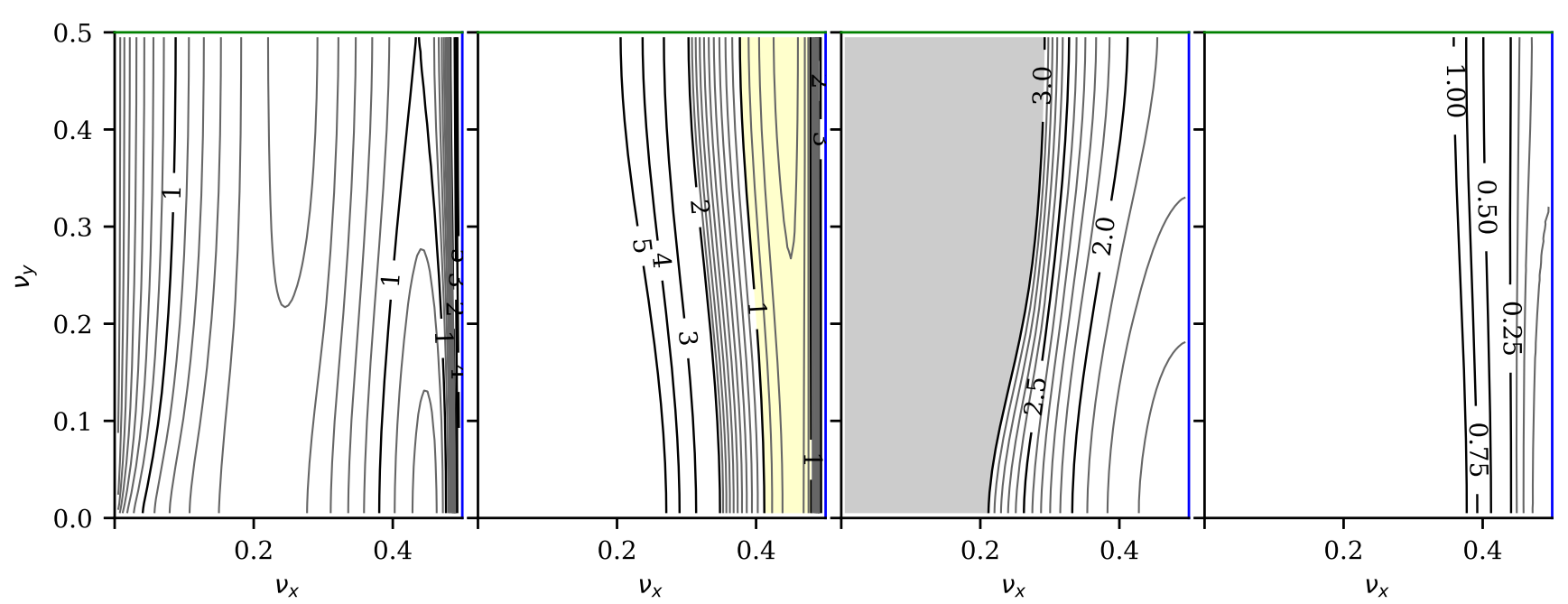

FIG. 8. From left to right: (1) optimal $G$ objective, (2) emittance ratio $F$ for $G$ objective, (3-4) quantities like in Fig. 4 (see legend therein) for optimal $G$.

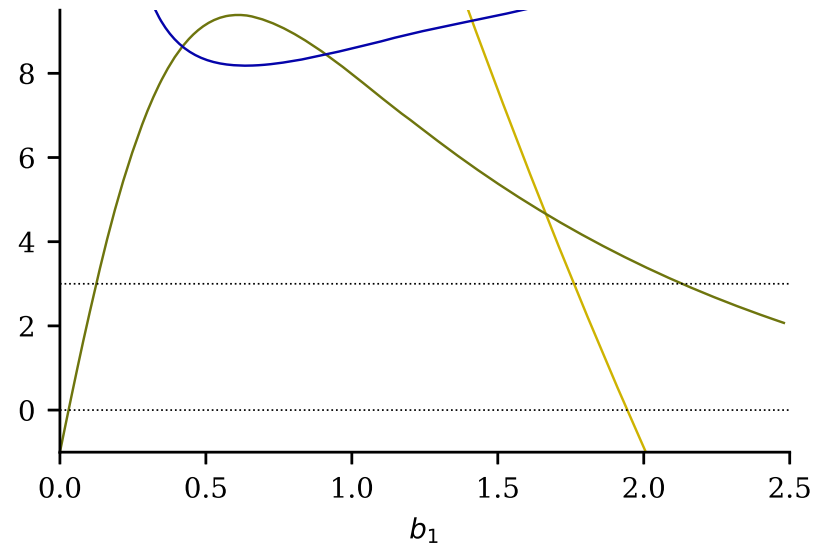

FIG. 9. Figures of merit in dependence of the dipole coefficient $b_{1}$ (see Fig. 5) for $\nu_{\mathrm{x}}=0.15, \nu_{\mathrm{y}}=0.35$.

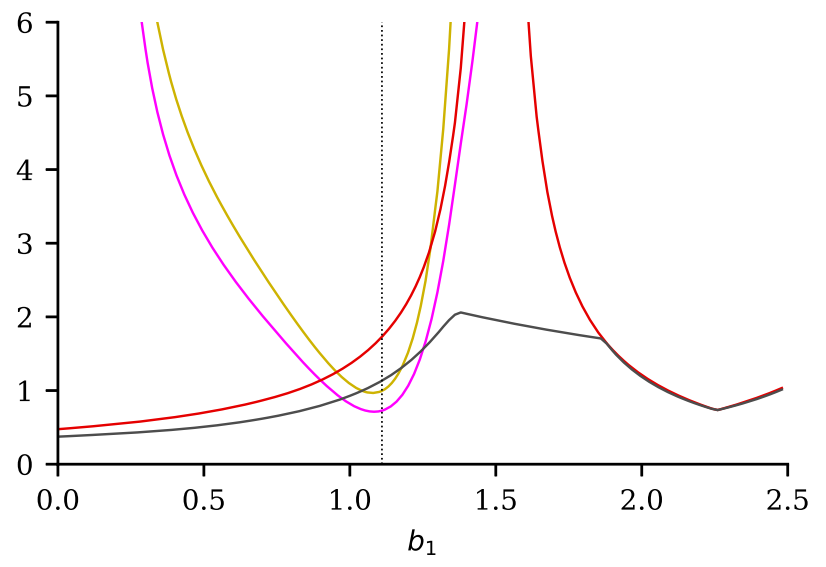

FIG. 10. $G$ objective for the standard sextupole harmonics ( $P=1$ : yellow) and extended harmonics $(P=2$ : magenta) as well as $\max |m|(P=1$ : red, $P=2$ : dark gray $)$ in dependence of the dipole coefficient $b_{1}$. The example value of $b_{1}$ is marked with a dashed line. harmonic coefficient $m_{2}$ is used to reduce max $|m|$ without changing optical functions.

To compensate chromaticity, we are required to solve a more general variant of Eq. (39)

$$
\vec{A}_{\mathrm{x}} \cdot \vec{m}=-\xi_{\mathrm{x}}, \quad \vec{A}_{\mathrm{y}} \cdot \vec{m}=\xi_{\mathrm{y}}
$$

with the components of $\vec{A}_{\mathrm{x}, \mathrm{y}}$ holding scaled Fourier components of $\tilde{\eta} \tilde{\beta}_{\mathrm{x}, \mathrm{y}}$. This system is underdetermined; its solution space in three dimensions is given as

$$
\vec{m}=\vec{m}_{(0)}+a\left(\vec{A}_{\mathrm{x}} \times \vec{A}_{\mathrm{y}}\right) \quad \text { for } a \in \mathbb{R}
$$

with $\vec{m}_{(0)}$ being an arbitrary solution. For our computation we use the least-squares solution of the system (44).

The quantity $\max |m|$ can be computed with minor effort, as we require it to be minimal under the constraint of full chromaticity compensation-this is achieved using an elementary optimization procedure on the scalar $a$.

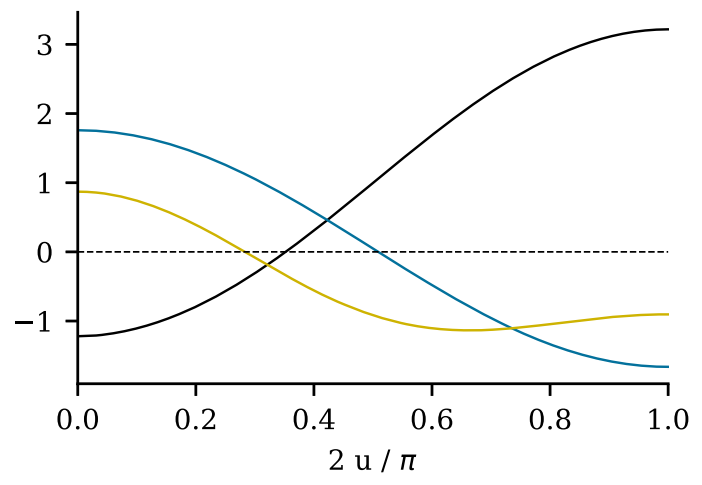

FIG. 11. Distribution of dipole (black), quadrupole (blue) and sextupole fields (yellow) for the example solution marked in Fig. 10. The optical functions are identical to those in Fig. 6. 


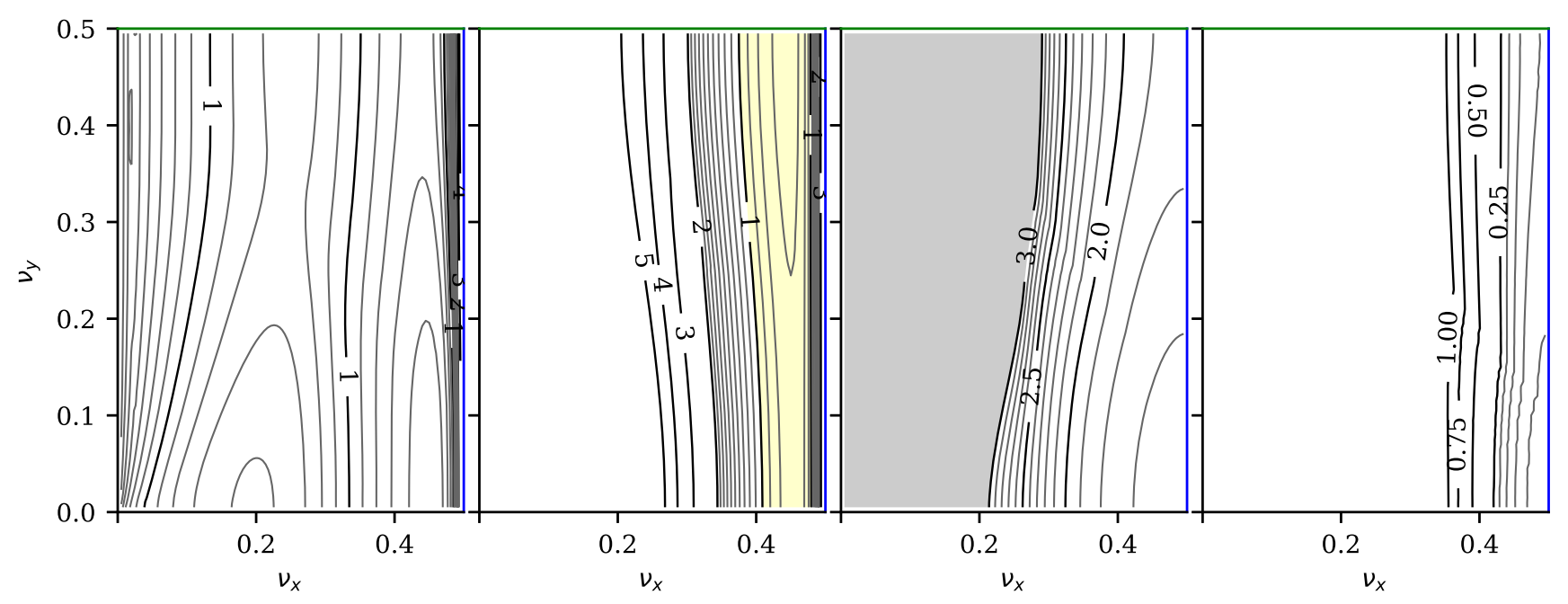

FIG. 12. Figures of merit for $G$-optimized solutions with extended sextupole harmonics $(P=2)$ in tune space. See legend in Fig. 8 .

The results of this optimization in tune space are shown in Fig. 12. Relative to the setup using just constant and fundamental harmonic $(P=1)$, an overall reduction of the $G$ objective has been achieved, reaching values $G<0.7$ in the low-emittance region.

This can be observed in more detail for our example tune $\nu_{\mathrm{x}}=0.45, \nu_{\mathrm{y}}=0.35$ in Figs. 10 and 11. The maximum value of $|m(u)|$ has been reduced by decreasing the sextupole strength at the position of maximum bending. This is reasonable as the large sextupolar fields at this location have a negligible influence on chromaticity compensation.

\section{Fringe effects}

The required magnetic potentials can always be constructed in principle, even when including fringe effects. This is discussed in the following in a compressed form, with Appendix $\mathrm{C}$ giving more details.

We approximate $(r, \phi, s)$ as a cylindrical coordinate system. Revisiting Eq. (1), we restrict the basis for $\Psi$

$$
\Psi=\sum_{n=1}^{3} \sum_{p=0}^{2} V_{n, p} \Psi_{n, p},
$$

to match the conditions of symmetry in the $s=0$ transverse plane, as well as symmetry in the $x$-s plane (only upright multipoles) by defining

$$
\Psi_{n, p}=-D_{n}\left(\bar{k}_{p}, r\right) \sin (n \phi) \cos \left(\bar{k}_{p} s\right) .
$$

One can obtain the field in the machine plane via the relations

$$
B_{y}(x) \equiv B_{\phi}(r=x, \phi=0), \quad B_{\phi}=-\frac{1}{r} \frac{\mathrm{d} \Psi}{\mathrm{d} \phi}
$$

By series expansion of $B_{y}(x, s)$ in $x$ (Appendix C), the dipolar and quadrupolar fields on the beam path are given by

$$
\begin{gathered}
B_{y}(0, s)=\sum_{p} V_{1, p} \cos \left(\bar{k}_{p} s\right) \\
\frac{d B_{y}}{d x}(0, s)=\sum_{p} V_{2, p} \cos \left(\bar{k}_{p} s\right) .
\end{gathered}
$$

Note that the linear vertical dependence of $B_{y}$ is also fixed to be that of a standard quadrupole field by the requirement of vanishing curl in $s$ direction. One can relate the $V$ coefficients to the normalized Mathieu cell coefficients using

$B_{y}(0, s)=(B \rho) / \rho(s), \quad \frac{d B_{y}}{d x}(0, s)=(B \rho) \kappa(s)$.

For the sextupolar fields, the relation is given by Eq. (36). Here, mixing with the $V_{1,1}$ components from the dipole potential occurs, as (Appendix C)

$$
\begin{aligned}
\frac{d^{2} B_{y}}{d x^{2}}(0, s)= & V_{3,0}+V_{3,1}(1+f) \cos \left(\bar{k}_{1} s\right) \\
& +V_{3,2} \cos \left(\bar{k}_{2} s\right),
\end{aligned}
$$

with the fringe factor

$$
f=-\left(\frac{\theta}{\pi}\right)^{2} \frac{b_{1}}{2 \bar{m}_{1}} .
$$

Here, $\bar{m}_{1}$ is the value of the $m_{1}$ sextupole coefficient when ignoring fringe effects. With fringe effects, the value of $m_{1}$ is shifted to $\mathrm{C}$ 


$$
m_{1}=\bar{m}_{1}(1+f) .
$$

As an estimate for the typical strength of the fringe effect, assuming $\left|b_{1}\right|,\left|m_{1}\right| \sim 1$, we can use the expression $(\theta / \pi)^{2}$ occurring in $f$. For bending angles per cell of $\theta \leq 5 \mathrm{deg}$, we obtain $(\theta / \pi)^{2} \leq 1 / 36^{2}<10^{-3}$.

For larger values of $|f|$, the sextupole strength can always be readjusted to yield the proper value of $m_{1}$. Note that with the fringe effect, $m_{1}$ does not refer to a standard sextupole in the transverse plane anymorehowever, we only require the field in the machine plane to compensate chromaticity via horizontal dispersion; the quadrupolar fields in the machine plane are always properly defined due to the condition of vanishing curl as stated.

\section{SLS 2.0 EXAMPLE}

The Swiss Light Source upgrade (SLS 2.0) has a unit cell length of $2.165 \mathrm{~m}$ and a unit cell bending angle of $5 \mathrm{deg}$. The average curvature radius and the characteristic magnetic field density from Eq. (21) are approximated using these values as

$$
1 /\langle 1 / \rho\rangle=24.81 \mathrm{~m}, \quad B_{\mathrm{c}}=322.5 \mathrm{mT} .
$$

According to Eq. (20) and assuming a normal-conducting magnet limit of $\max B \sim 2 \mathrm{~T}$, we get $\max |b| \sim 6.2$, or $\max \left|b_{1}\right| \sim 2.6$.

We assume the maximum applicable sextupole strength at $\max |\mu|=650 \mathrm{~m}^{-3}$, which is a conservative estimate consistent with the present lattice design. By using Eq. (41) we are able to compute the optimal cell length for a Mathieu cell with example parameters for SLS 2.0. Using the standard sextupole harmonics $(P=1$, Table I) we obtain $\max |m| \sim 1.726$, resulting in an optimal cell length of $\sim 1.592 \mathrm{~m}$. Using the extended sextupole harmonics $(P=2$, Fig. 10) we obtain a reduced value of $\max |m| \sim 1.134$, resulting in an optimal cell length of $\sim 1.433 \mathrm{~m}$.

\section{A. Improved optimal cell length estimate using pole-tip fields}

It should be noted that, due to the overlapping of fields with different multipolar order, the pole tip field of a combined-function magnet will be higher than that of the sextupole component, thus increasing the optimal cell length. For a detailed example we calculate pole-tip fields $B_{r}^{\mathrm{pt}}$ with the common approach [29], i.e., without considering longitudinal variation as in Eq. (1), as

$$
\frac{B_{r}^{\mathrm{pt}}(s, \phi, R)}{(B \rho)}=\frac{\sin \phi}{\rho(s)}+\sin (2 \phi) \kappa(s) R+\sin (3 \phi) \mu(s) R^{2}
$$

with the pole-tip radius $R$, or as a unitless equation,

$$
\begin{aligned}
\frac{B_{r}^{\mathrm{pt}}}{B_{\mathrm{c}}}(u, \phi, R)= & \sin \phi b(u)+\sin (2 \phi) k(u)\left(\frac{L_{\mathrm{c}}}{L}\right)^{2} \\
& +\sin (3 \phi) m(u)\left(\frac{L_{\mathrm{c}}}{L}\right)^{4}
\end{aligned}
$$

where we defined the characteristic length

$$
L_{\mathrm{c}}=\pi \sqrt{R /\langle 1 / \rho\rangle}
$$

containing the geometric mean of chamber and average curvature radius. In the case of interest, the maximum poletip field strength is not dominated by $m(s)$ alone, as would be the case for $L_{\mathrm{c}} / L \gg 1$. Instead, the situation $L_{\mathrm{c}} \sim L$ occurs because multipoles of different order often have comparable pole-tip field magnitudes.

While the pole-tip field can be used as an estimate for the technical feasibility of magnet design, this estimate can be improved further. To do so, we take into account the empirical knowledge that the feasible pole-tip fields decrease with the multipole order $n-$ e.g., for the SLS 2.0 separate-function magnets we may assume an inverse relation $\max B_{r}^{\mathrm{pt}} \sim 2 \mathrm{~T} / n$.

To include the improved estimate for combined-function magnets, their contributions from Eq. (57) are weighted with their order, leading to the definition of a weighted pole-tip field via

$$
\begin{aligned}
\frac{B_{r}^{\mathrm{w}}}{B_{\mathrm{c}}}(u, \phi, R)= & \sin \phi b(u)+2 \sin (2 \phi) k(u)\left(\frac{L_{\mathrm{c}}}{L}\right)^{2} \\
& +3 \sin (3 \phi) m(u)\left(\frac{L_{\mathrm{c}}}{L}\right)^{4}
\end{aligned}
$$

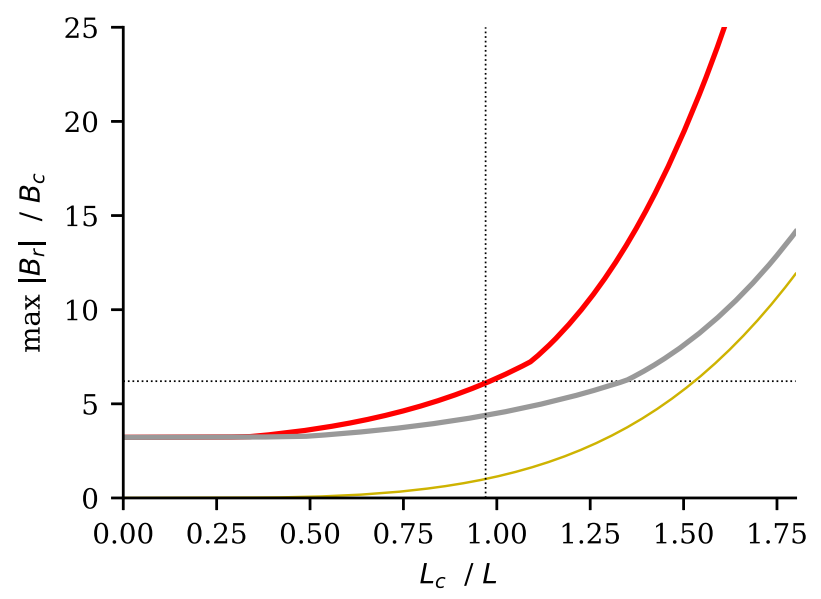

FIG. 13. Maximum weighted pole-tip field $B_{r}^{\mathrm{w}}$ (red), actual pole-tip field $B_{r}^{\mathrm{pt}}$ (gray), and pole-tip field of sextupole component (yellow) in dependence of normalized inverse cell length $L_{\mathrm{c}} / L$, all in units of characteristic field density and length. The values of $\max \left|B_{r}\right|=2 \mathrm{~T}$ for SLS 2.0 assumptions and the corresponding value of $L_{\mathrm{c}} / L$ for a maximum weighted poletip field close to that strength are denoted by dotted lines. 


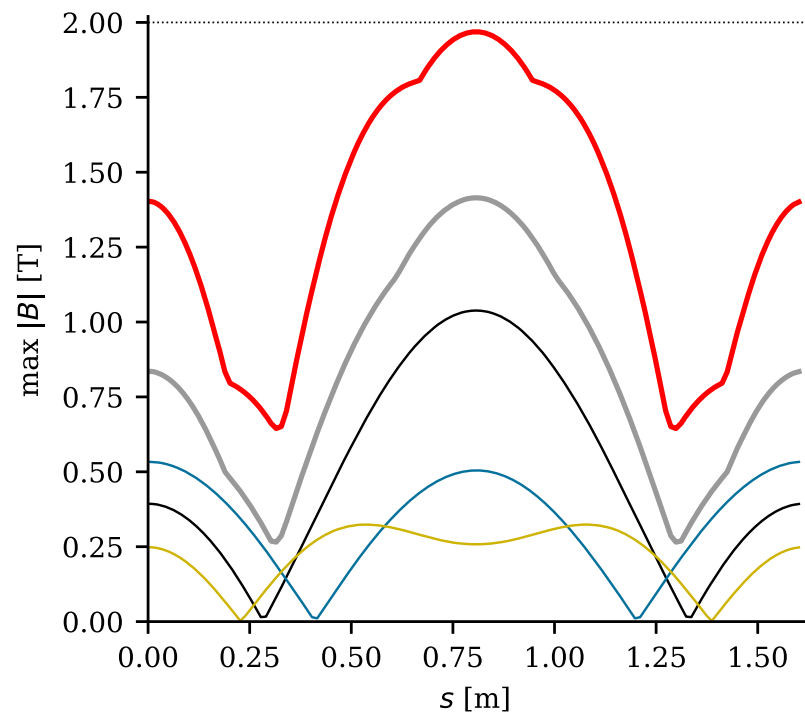

FIG. 14. Absolute maxima of dipole (black), quadrupole (blue) and sextupole (yellow) pole-tip fields, actual pole-tip field $B_{r}^{\mathrm{pt}}$ (gray), and weighted pole-tip field $B_{r}^{\mathrm{W}}$ (red) along $s$ for the SLS 2.0 example (cf. Fig. 15).

We can obtain good approximations of the maximum pole-tip fields for a given value of $L_{\mathrm{c}} / L$ by computing the maximum value of $B_{r} / B_{\mathrm{c}}$ on a grid of $(\phi, u)$ points. In this work we use 128 values of $u$ and 16 values of $\phi$. The result of this computation with the example cell is shown in Fig. 13. One can observe that, as expected, the sextupole strengths dominate for large $L_{\mathrm{c}} / L$; small values are dominated by the constant dipole contribution.

\section{B. SLS 2.0 parameters and results}

For SLS 2.0 we assume a chamber radius $R=10 \mathrm{~mm}$ and obtain the characteristic length $L_{\mathrm{c}} \sim 1.565 \mathrm{~m}$. The technical limit of pole-tip fields in such a distributed magnet structure is yet to be determined. Comparing the actual pole-tip field in Fig. 13 with the sextupole-only contribution, we can see that the optimal cell length increases significantly when all multipoles are considered.

We now consider the example values marked in Fig. 13, where the optimal cell length is $L \sim 1.031 L_{\mathrm{c}} \sim 1.614 \mathrm{~m}$ and $\max \left|B_{r}^{\mathrm{w}}\right|$ is close to $2 \mathrm{~T}$ with a small safety margin. The distribution of multipole contributions to the pole-tip fields is shown in Fig. 14.

This example magnet configuration is analyzed using the optics code OPA [29]. As optics codes usually do not work in Fourier space, we discretize the solution into segments of dipole-quadrupoles and thin sextupoles. For convenience, we choose 128 segments for each magnet type.

The optics results are shown in Fig. 15, and Table II shows global figures of merit as computed by OPA. For the betatron tunes, we can observe that for our example, neglecting weak focusing and edge focusing as stated in Sec. IV is justified. Within the assumptions about pole-tip fields, which may exceed technical limits, and our assumptions about weak focusing and general feasibility of the nontrivial magnetic field arrangement, we obtain an emittance of $\sim 33.2 \mathrm{pm}$, which is significantly less than the SLS 2.0 design of $\sim 100 \mathrm{pm}$ [30]. The Mathieu cell's horizontal damping partition is in the range of SLS 2.0 designs with the present $J_{x}$ also being $\sim 1.8$ in difference to earlier designs [30]. The $\sim 760 \mathrm{keV}$ energy loss per turn is slightly more than the present SLS 2.0 design at $\sim 690 \mathrm{keV}$, while the damping times of both the Mathieu cell and the SLS 2.0 unit cell are in the order of a few ms.

In addition to the aforementioned complications, the cell is almost isochronous with a momentum compaction in the $10^{-6}$ range. This can be circumvented by a minor decrease of $b_{1}$ at the expense of slightly increased emittance (see Fig. 5).

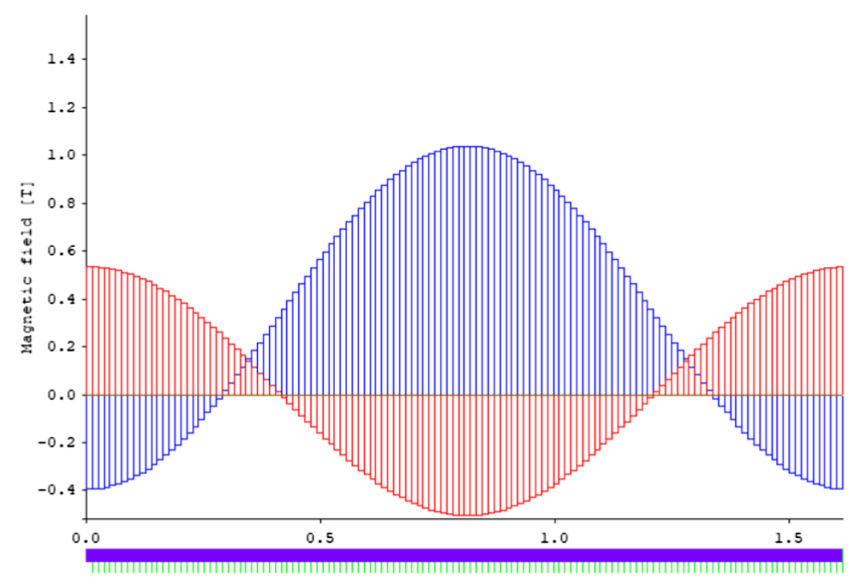

(a)

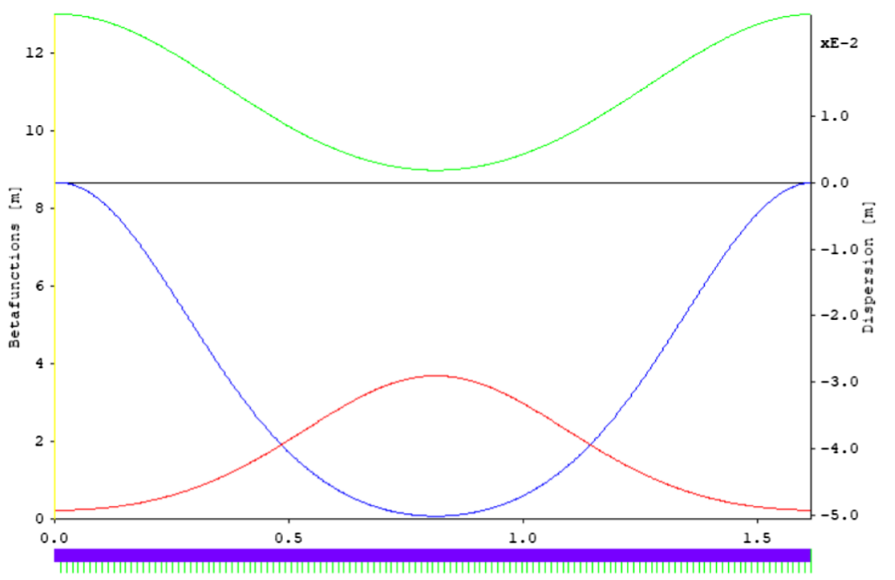

(b)

FIG. 15. Properties of the Mathieu cell for the SLS 2.0 example as computed by OPA. (a) Pole-tip fields for multipole slices (cf. Fig. 14). Only fields for multipoles of finite length (dipole: blue, quadrupole: red) are shown. (b) optical functions of optimized Mathieu cell for SLS 2.0 in opa $\left(\beta_{\mathrm{x}}\right.$ : blue, $\beta_{\mathrm{y}}$ : red, $\eta$ : green). 
TABLE II. Lattice parameters of the Mathieu cell for the SLS 2.0 example as computed by OPA. Rounded values have been used where appropriate.

\begin{tabular}{lc}
\hline \hline Parameter & Value \\
\hline Cell length & $1.6140 \mathrm{~m}$ \\
Horizontal cell tune $\nu_{\mathrm{x}}$ & 0.45057 \\
Vertical cell tune $\nu_{\mathrm{y}}$ & 0.34988 \\
Natural horizontal cell chromaticity $\xi_{\mathrm{x}}$ & -1.84995 \\
Natural vertical cell chromaticity $\xi_{\mathrm{y}}$ & -0.67107 \\
Momentum compaction & $-2.718 \times 10^{-6}$ \\
Horizontal damping partition $J_{\mathrm{x}}$ & 1.8207 \\
Beam energy & $2.4 \mathrm{GeV}$ \\
Radiated energy/cell passage & $4.243 \mathrm{keV}$ \\
Natural energy spread & $8.6422 \times 10^{-4}$ \\
Horizontal damping time & $3.345 \mathrm{~ms}$ \\
Vertical damping time & $6.090 \mathrm{~ms}$ \\
Longitudinal damping time & $5.164 \mathrm{~ms}$ \\
Horizontal emittance $\epsilon_{\mathrm{x}}$ & $33.19 \mathrm{pm}$ \\
$I_{1}$ integrated over cell & $-4.387 \times 10^{-6} \mathrm{~m}$ \\
$I_{2}$ integrated over cell & $9.084 \times 10^{-3} \mathrm{~m}^{-1}$ \\
$I_{3}$ integrated over cell & $9.467 \times 10^{-4} \mathrm{~m}^{-2}$ \\
$I_{4}$ integrated over cell & $-7.455 \times 10^{-3} \mathrm{~m}^{-1}$ \\
$I_{5}$ integrated over cell & $6.495 \times 10^{-8} \mathrm{~m}^{-1}$ \\
\hline \hline
\end{tabular}

\section{CONCLUSION}

In this work, we introduced Mathieu unit cells as elementary approximations for periodic lattice systems. Due to their distributed multipolar structure, they allow for the inclusion of combined-function effects, as well as the computation of common figures of merit like momentum compaction and emittance. They even predict the usefulness of combining longitudinal gradients with reverse bending, and reach sub-TME emittance.

By its nature, the goal of this study can only be to illuminate an "undercurrent" of the sinusoidal focusing concept, permeating lattice design, that otherwise is concealed by practical requirements of accelerator technology. It goes without saying that a realizable lattice design requires detailed studies incorporating a multitude of boundary conditions [28,31] e.g., dynamic aperture considerations, robustness in case of field deviations $[32,33]$, which depend on the cell length in a nontrivial manner.

Mathieu cells are useful tools for investigating basic lattice configurations and performance limits. Sinusoidal bending forces are commonly used in the description of undulator fields (see, e.g., [14]). In the context of further progress on MBA miniaturization and combined-function magnet lattices, e.g., [34-37], the concept of Mathieu cells could thus help to shape future lattice designs.

The source code for all computations in this work, excluding the ones performed in OPA, is based on the SciPy framework [38,39] and fully accessible [40].

\section{ACKNOWLEDGMENTS}

The author thanks M. Kranjčević, J. Kallestrup, A. Streun, and J. Bengtsson for improving the manuscript by proofreading and/or hinting at useful references. Furthermore, the author appreciates the general support of M. Aiba, M. Böge, J. Chrin, and T. Schietinger.

\section{APPENDIX A: PROPERTIES OF SCALED BESSEL FUNCTION}

Using the series expansion of $I_{n}$ [41]

$$
I_{n}(x)=(x / 2)^{n} \sum_{q=0}^{\infty} \frac{(x / 2)^{2 q}}{q !(n+q) !}
$$

and the definition

$$
D_{n}(k, x)=I_{n}(k x) /(k / 2)^{n},
$$

one obtains

$$
D_{n}(k, x)=x^{n} \sum_{q=0}^{\infty}(k / 2)^{2 q} \frac{x^{2 q}}{q !(n+q) !} .
$$

Note that a removable singularity exists at $k=0$,

$$
\lim _{k \rightarrow 0} D_{n}(k, x)=x^{n} / n !
$$

The derivative, required for the radial component of field density, can be expressed as a series in $k$,

$$
\begin{aligned}
\frac{\mathrm{d} D_{n}(k, x)}{\mathrm{d} x} & =x^{n-1} \sum_{q=0}^{\infty}(2 q+n) \frac{x^{2 q}}{q !(n+q) !} \\
& =n \frac{x^{n-1}}{n !}+(2+n)\left(\frac{k}{2}\right)^{2} \frac{x^{n+1}}{(n+1) !}+\ldots \\
& =\frac{x^{n-1}}{(n-1) !}\left[1+k^{2} \frac{n+2}{4 n(n+1)} x^{2}+\cdots\right] .
\end{aligned}
$$

\section{APPENDIX B: SCALING CELL LENGTH IN A FIXED ARC}

To obtain results as general as possible, this work often uses multipoles normalized to a dimensionless unit cell. A rule is that the standard, rigidity-normalized multipole fields $-1 / \rho$ for curvature, $\kappa$ for quadrupole focusing strength, $\mu$ for sextupole strength-are denoted by Greek letters. Their cell-normalized, dimensionless counterparts $-b$ for normalized curvature, $k$ for normalized quadrupole, $m$ for normalized sextupole strength, are denoted by Latin letters.

The dimensionless, cell-normalized optics functions are also denoted with a tilde, $\tilde{\boldsymbol{}}$, to distinguish them from the 
standard optics functions. All occurring synchrotron integrals $I$. are cell-normalized.

When replacing the path length $s$ by a scaled path length $u=\pi s / L$, we require the scaled solution $x(u)$ to fulfill Hill's equation (4)

$$
\frac{\mathrm{d}^{2}}{\mathrm{~d} s^{2}} x(u)+\kappa(u) x(u)=0
$$

so that

$$
\frac{\mathrm{d}^{2}}{\mathrm{~d} u^{2}} x(u)+\left[(L / \pi)^{2} \kappa(u)\right] x(u)=0
$$

and by comparison

$$
\kappa(u)=\left(\frac{\pi}{L}\right)^{2} k(u)
$$

resulting in the standard quadrupole strength scaling with the inverse square of cell length.

Since we require the tune for all cells to be independent of the cell length, this should also apply to the natural chromaticity so that $\int \beta \kappa \mathrm{d} s \propto \beta / L$ is constant, and

$$
\beta(u)=\frac{L}{\pi} \tilde{\beta}(u)
$$

is linear in $L$, so $\int \tilde{\beta} k \mathrm{~d} s$ is also constant, with $\tilde{\beta}$ being the cell-normalized optics function.

Furthermore, the dispersion function $\eta(s)$ must fulfill the inhomogeneous Hill's equation

$$
1 / \rho(u)=\frac{\mathrm{d}^{2}}{\mathrm{~d} s^{2}} \eta(u)+\kappa(u) \eta(u)
$$

As the average arc curvature should remain constant, we require $b(u)$ to be independent of cell length. Division by $\langle 1 / \rho\rangle$ yields

$$
\begin{aligned}
b(u) & =\frac{\mathrm{d}^{2}}{\mathrm{~d} s^{2}} \frac{\eta(u)}{\langle 1 / \rho\rangle}+\kappa(u) \frac{\eta(u)}{\langle 1 / \rho\rangle} \\
& =\frac{\mathrm{d}^{2}}{\mathrm{~d} u^{2}} \tilde{\eta}(u)+k(u) \tilde{\eta}(u)
\end{aligned}
$$

with

$$
\tilde{\eta}(u)=\left(\frac{\pi}{L}\right)^{2} \frac{\eta(u)}{\langle 1 / \rho\rangle} .
$$

For the compensated chromaticity to be independent of cell length, we require

$$
\begin{aligned}
\int \beta(u) \eta(u) \mu(u) \mathrm{d} s & =\left(\frac{L}{\pi}\right)^{4}\langle 1 / \rho\rangle \int \tilde{\beta}(u) \tilde{\eta}(u) \mu(u) \\
& =\text { const. }
\end{aligned}
$$

Then sextupole strength scales as

$$
\mu(u)=\left(\frac{\pi}{L}\right)^{4} \frac{m(u)}{\langle 1 / \rho\rangle} .
$$

Note that this inverse quartic scaling is due to the average curvature $\langle 1 / \rho\rangle$ remaining constant-if the ring was miniaturized as a whole, $\langle 1 / \rho\rangle \propto 1 / L$ would hold, resulting in inverse cubic scaling and corresponding to the multipole order.

\section{APPENDIX C: FIELDS IN THE MACHINE PLANE}

We are interested in the field density in the machine plane, given by Eq. (48)

$$
B_{y}(x, s)=\sum_{n=1}^{3} \sum_{p=0}^{2} V_{n, p} \frac{D_{n}\left(\bar{k}_{p}, x\right)}{x} n \cos \left(\bar{k}_{p} s\right),
$$

specifically its series expansion in $x$, yielding the multipole components. Inserting Eq. (A3) into Eq. (C1), one obtains an approximation for small $x$ as

$$
\begin{aligned}
B_{y}(x, s)= & \sum_{p=0}^{2} \cos \left(\bar{k}_{p} s\right) . \\
& \cdot\left[V_{1, p}+V_{2, p} x+\left(V_{3, p}+V_{1, p} \bar{k}_{p}^{2} / 4\right) \frac{x^{2}}{2}+O\left(x^{3}\right)\right] .
\end{aligned}
$$

Utilizing that $\bar{k}_{0}=0$, and that the dipolar and quadrupolar fields are set to zero for the $p=2$ harmonic $\left(V_{3,1}=V_{3,2}=0\right)$, we can simplify this series to

$$
\begin{aligned}
& B_{y}(x, s) \\
& =V_{1,0}+V_{1,1} \cos \left(\bar{k}_{1} s\right)+\left[V_{2,0}+V_{2,1} \cos \left(\bar{k}_{1} s\right)\right] x \\
& +\left[V_{3,0}+\left(V_{3,1}+V_{1,1} \frac{\bar{k}_{1}^{2}}{4}\right) \cos \left(\bar{k}_{1} s\right)+V_{3,2} \cos \left(\bar{k}_{2} s\right)\right] \frac{x^{2}}{2} \\
& \quad+O\left(x^{3}\right) .
\end{aligned}
$$

For almost all coefficients $V$, we find that each is proportional to a dipolar, quadrupolar, or sextupolar focusing term. The only exception is the quadratic coefficient

$$
\left(V_{3,1}+V_{1,1} \frac{\bar{k}_{1}^{2}}{4}\right)
$$


feeding up the dipolar fringe effect via the $V_{1,1}$ coefficient. To quantify its effect, we define a fringe factor

$$
f=\frac{\bar{k}_{1}^{2}}{4} \frac{V_{1,1}}{V_{3,1}}=\frac{\pi^{2}}{L^{2}} \frac{V_{1,1}}{V_{3,1}}
$$

so that

$$
\begin{aligned}
\frac{d^{2} B_{y}}{d x^{2}}(0, s)= & V_{3,0}+V_{3,1}(1+f) \cos \left(\bar{k}_{1} s\right) \\
& +V_{3,2} \cos \left(\bar{k}_{2} s\right)
\end{aligned}
$$

And, by using Eq. (36) and Eq. (B9),

$m(u)=m_{0}+2 \bar{m}_{1}(1+f) \cos (2 u)+2 m_{2} \cos (4 u)$,

with $\bar{m}_{1}$ being the sextupole coefficient set when ignoring the fringe effect $(f=0)$, and

$$
m_{1}=\bar{m}_{1}(1+f) .
$$

Again by connecting the $V$ coefficients in $f$ to the $b$ and $m$ components via Eq. (36) and Eq. (B9), one obtains

$$
f=-\left(\frac{L\langle 1 / \rho\rangle}{\pi}\right)^{2} \frac{b_{1}}{\bar{m}_{1}}=-\left(\frac{\theta}{\pi}\right)^{2} \frac{b_{1}}{2 \bar{m}_{1}} .
$$

[1] R. Chasman, G. K. Green, and E. M. Rowe, Preliminary design of a dedicated synchrotron radiation facility, IEEE Trans. Nucl. Sci. 22, 1765 (1975).

[2] G. Vignola, Preliminary design of a dedicated $6 \mathrm{GeV}$ synchrotron radiation storage ring, Nucl. Instrum. Methods Phys. Res., Sect. A 236, 414 (1985).

[3] D. Einfeld and M. Plesko, A modified qba optics for low emittance storage rings, Nucl. Instrum. Methods Phys. Res., Sect. A 335, 402 (1993).

[4] M. Sommer, Optimization of the Emittance of electrons (positrons) storage rings, Tech. Rep. No. LAL/RT/83-15 (Laboratoire de l'Accélérateur Linéaire, 1983).

[5] L. C. Teng, Minimum emittance Lattice for Synchrotron Radiation Storage Rings, Tech. Rep. No. LS-17 (Argonne National Laboratory, 1985).

[6] R. Nagaoka and A. F. Wrulich, Emittance minimisation with longitudinal dipole field variation, Nucl. Instrum. Methods Phys. Res., Sect. A 575, 292 (2007).

[7] A. Streun and A. Wrulich, Compact low emittance light sources based on longitudinal gradient bending magnets, Nucl. Instrum. Methods Phys. Res., Sect. A 770, 98 (2015).

[8] M. A. D. Martinez, F. Toral, H. Ghasem, P. S. Papadopoulou, and Y. Papaphilippou, Longitudinally variable field dipole design using permanent magnets for clic damping rings, IEEE Trans. Appl. Supercond. 28, 1 (2018).
[9] J. P. Delahaye and J. P. Potier, Reverse bending magnets in a combined function lattice for the clic damping ring, in Proc. 1989 Particle Accelerator Conf., Chicago (1989) p. 1611.

[10] E. D. Courant, Accelerators, colliders, and snakes, Annu. Rev. Nucl. Part. Sci. 53, 1 (2003).

[11] A. Streun, The anti-bend cell for ultralow emittance storage ring lattices, Nucl. Instrum. Methods Phys. Res., Sect. A 737, 148 (2014).

[12] B. Riemann and A. Streun, Low emittance lattice design from first principles: Reverse bending and longitudinal gradient bends, Phys. Rev. Accel. Beams 22, 021601 (2019).

[13] T. Zhang and X. Huang, Numerical optimization of a low emittance lattice cell, Nucl. Instrum. Methods Phys. Res., Sect. A 923, 55 (2019).

[14] K. Wille, The Physics of Particle Accelerators (Oxford University Press, New York, 2000).

[15] N. Christofilos, Focussing system for ions and electrons, (1950), US Patent 2736799A.

[16] J. D. Jackson, Classical Electrodynamics, 3rd ed. (Wiley, New York, 1999).

[17] G. W. Hill, On the part of the motion of the lunar perigee which is a function of the mean motions of the sun and moon, Acta Math. 8, 1 (1886).

[18] E. D. Courant and H. S. Snyder, Theory of the alternatinggradient synchrotron, Ann. Phys. (N.Y.) 281, 1 (1958).

[19] J.E. Sträng, On the characteristic exponents of Floquet solutions to the Mathieu equation, Acad. Roy. Belg. Bull. Cl. Sci. 16, 269 (2005), arXiv:math-ph/0510076.

[20] Carmen Chicone, Ordinary Differential Equations with Applications (Springer, New York, 2006).

[21] S. A. Wilkinson, N. Vogt, D. S. Golubev, and J. H. Cole, Approximate solutions to Mathieu's equation, Phys. E 100, 24 (2018).

[22] H. Wiedemann, Particle Accelerator Physics, 4th ed. (Springer, New York, 2015).

[23] B. Riemann, The bilinear-exponential closed-orbit model and its application to Storage Ring beam diagnostics, Ph.D. thesis, TU Dortmund University, 2016, chapter 2.

[24] R. H. Helm, M. J. Lee, P. L. Morton, and M. Sands, Evaluation of synchrotron radiation integrals, IEEE Trans. Nucl. Sci. 20, 900 (1973).

[25] M. Sands, The Physics of Electron Storage Rings: An Introduction, Tech. Rep. No. R-121 (SLAC, 1979) with addendum.

[26] S. M. Liuzzo, D. Einfeld, L. Farvacque, and P. Raimondi, Hybrid Multi Bend Achromat at $3 \mathrm{GeV}$ for future 4th generation light sources, in Proc. 7th Int. Particle Accelerator Conf. (IPAC'16), Busan (JACoW, Geneva, 2016), p. 2822.

[27] J. Bengtsson, Ultra Low Emittance Light Sources, in Proc. 11th European Particle Accelerator Conference, Genoa, Italy (EPS-AG, Genoa, Italy, 2008), p. 988.

[28] Y. Cia, W. Chou et al., ICFA Beam Dynamics Newsletter 57: Low emittance rings (2012), https://icfa-usa.jlab.org/ archive/newsletter.shtml.

[29] OPA accelerator optics software, https://ados.web.psi.ch/ opa (2017).

[30] A. Streun, T. Garvey, L. Rivkin, V. Schlott, T. Schmidt, P. Willmott, and A. Wrulich, SLS-2 - the upgrade of the Swiss Light Source, J. Synchrotron Radiat. 25, 631 (2018). 
[31] P. Emma and T. Raubenheimer, Systematic approach to damping ring design, Phys. Rev. Accel. Beams 4, 021001 (2001).

[32] J. Bengtsson, Robust design of low emittance rings, in Low Emittance Rings workshop 2014, Frascati (2014).

[33] J. Bengtsson and A. Streun, Robust Design Strategy for SLS-2, Paul Scherrer Institut Tech. Report No. SLS2BJ84-001, 2017.

[34] A. V. Bogomyagkov, E. B. Levichev, and S. V. Sinyatkin, Weak focusing low emittance storage ring with large $6 \mathrm{~d}$ dynamic aperture based on canted cosine theta magnet technology, arXiv:1906.09692.

[35] P. T. Tavares, Long term plans at MAX IV, in 2nd workshop on low emittance ring lattice design (2016).

[36] P. Yang, Z. Bai, T. Zhang, D. Xu, and L. Wang, Design of a hybrid ten-bend-achromat lattice for a diffraction-limited storage ring light source, Nucl. Instrum. Methods Phys. Res., Sect. A 943, 162506 (2019).

[37] G. Wang, T. Shaftan, V. Smaluk, Y. Hidaka, O. Chubar, T. Tanabe, J. Choi, S. Sharma, C. Spataro, and N. A. Mesentsev, Complex bend. II. A new optics solution, Phys. Rev. Accel. Beams 22, 110703 (2019).

[38] P. Virtanen, R. Gommers, T. E. Oliphant et al., SciPy 1.0Fundamental Algorithms for Scientific Computing in Python, arXiv:1907.10121.

[39] J. D. Hunter, Matplotlib: A 2D graphics environment, Comp. Sci. Eng. 9, 90 (2007).

[40] B. Riemann, Elementary computations for studying Mathieu unit cells, (2020), https://github.com/b-riemann/mathieu-cell.

[41] M. Abramovitz and I. Stegun, Handbook of Mathematical Functions, 10th ed. (National Bureau of Standards, Gaithersburg, Maryland, U.S.A., 1972). 